\title{
Develope An Certificate Authority Based Encryption Cryptography Model For Mobile Applications In Cloud Computing
}

\author{
${ }^{1}$ Dr.M.Mahalakshmi ${ }^{2}$ Mr. S.Kalyanasundaran \\ 1,2 School of Information Science, Gondar University ,Ethiopia
}

\begin{tabular}{lrr}
\multicolumn{3}{l}{ Article Information } \\
Received & $:$ & 16 March 2020 \\
Revised & $:$ & 21 March 2020 \\
Accepted & $:$ & 25 March 2020 \\
Published & $:$ & 10 April 2020
\end{tabular}

Corresponding Author:

Dr. M. Mahalakshmi

Email:maha32@yahoo.com

\begin{abstract}
The backbone of this research is to design and developed an effective cloud environment for pretending and protection of portable client device information put away in the cloud. Portable gadgets, for example, advanced mobile phones or tablets that are fundamentally more restricted than PCs as far as memory, processors, secured operating system, and accessible system data transmission bandwidth because of that all information migrate in to cloud environment. A portable client may go about as an information owner will demand key from the cloud administrator, scrambles the information and afterward it transfers the information in the cloud. In the cloud secured encryption is finished. Certificate Authority Based Encryption (CABE) approach is utilized for security and adaptability. Frequent monitoring is accomplished for Privacy, Security, trustworthiness and accessibility.
\end{abstract}

Keywords: CABE approach, cloud environment, cloud manager, cloud monitoring, Portable devices.

Copyright () 2020: Dr. Mahalakshmi. This is an open access distribution, and reproduction in any medium, provided Access article distributed under the Creative Commons Attribution License the original work is properly cited License, which permits unrestricted use.

Citation: Dr. Mahalakshmi. "Develope a Certificate Authority Based Encryption Cryptography Model for Mobile Applications in Cloud Computing, "Journal of Science, Computing and Engineering Research, 1(1), 16-21, Mar-Apr 2020.

\section{INTRODUCTION}

In Mobile Cloud computing environment at its most straightforward infrastructure, alludes to a foundation where both the information storage and information processing occur outside of the mobile phone applications. Versatile cloud applications move the devices forcefully and information processing from the cell phones and into ground-breaking and brought together figuring stages situated in fogs, which are then gotten to over the remote association dependent on a client. Today mobile phones are utilized with rich cloud benefits by incorporating applications that consume web based services. These cloud administrations are conveyed in cloud. There are a few Smartphone working frameworks accessible, for example, Google's Android, Apple's iOS, RIM BlackBerry, Symbian, and Windows Mobile Phone. It should likewise be perceived that the ongoing pattern in modern cloud computing applications is for cloud information to be gotten to essentially by benefit appreciative cell phones, a training known as portable distributed computing. This applications classification incorporates clients of cell phones and tablets; in certain applications, it is fitting to think about keen remote sensors in a similar blend. Subsequently, any resolution giving extra security must not add difficult expenses to a portable client; explicitly, the quantity of transmissions must be limited to save the battery and overthe-air information utilization charges, and the measure of calculation should likewise be limited to avoid from adding noteworthy rearrangements to the client encounter while further weakening battery life. Another significant necessity is for information to be addressable with fine-grained get to controls on the record-level or better, to give adaptability. A single user sign in is to a great extent lacking in the present complex information recovery assignments.

Putting away information in a cloud infrastructure is done in secure style. The cloud service providers can't peruse put away information. The convention protocol is intended to be effective for asset obliged portable clients. An improvement is made over a conventional trait based encryption conspire. Improve the effective security is done through Certificate Authority Based Encryption in the cloud service provider. This further security measure is a significant variant suitable to highly sensitive data subject to recurrent access. Certificate Authority Based Encryption, as a method of converting the stored cipher text, it does not require elimination of features and ensuing key regeneration, and may be managed by a trusted authority without contribution of the data owner. Certificate Authority Based Encryption show of the proposed system is established on commercially popular mobile and cloud platforms. Auditing is provided for integrity and availability of the data in the cloud. 
In Section 2, literature survey on key management to secure cloud data storage is presented, with a focus on Certificate Authority Based Encryption in the context of applications accessed by mobile devices. In Section 3, a system model encompassing a mobile cloud computing system is presented. In Section 4, the proposed algorithm for Certificate Authority Based Encryption suitable for mobile users of the cloud is presented. In Section 5, the results of the implementation of Certificate Authority Based Encryption scheme on actual mobile devices and an operational cloud system are presented and discussed. Section 6 , provides concluding remarks and the future work.

\section{RELATED WORKS}

$\mathrm{ABE}$ and proxy re-encryption are merged together in another related work, fine-grained access control of resources are allowed while offloading Certificate Authority Based Encryption activity to the cloud provider [16]. It's various differences to the scheme that will be proposed. The info owner is involved in generating a key for every new user that joins or leaves the system, instead of offloading this task; it's not only a prohibitive cost for a mobile user, but also impractical thanks to the user's mobility. Another difference is that a secret key must be regenerated and redistributed for every user, in lazy fashion, whenever user revocation occurs, instead of allowing users to upgrade a standard group key, which reduces the communication cost and leads to higher efficiency. Furthermore, the reencryption occurs thanks to attribute redefinition and therefore the scheme is predicated on key-policy attributebased encryption (KP-ABE) and not CP-ABE, where the cipher text is related to a policy. A multi authority system has been proposed [17] that uses attribute-based access control to avoid the only point of failure of one key authority, and relies upon the info owner communicating with attribute authorities to get multiple encryption keys for hosted content.

The costs of communicating with multiple authorities and storing multiple keys could become prohibitive for mobile users. During a related work [18], each user submits multiple secret keys issued by authorities to a server to get a decryption token for every cipher text that's utilized in concert with the user's global secret key to perform a decryption. An equivalent costs related to multiple authorities apply, however, and such authorities complicate and increase the expense of system engineering. The authors are unaware of an identical scheme where fine- grained operations in attribute-based cryptography are reassigned across system components to attenuate the workload of mobile users; nor are other techniques found with performance benchmarked on commercial mobile and cloud systems, as herein, useful in assessing real-world viability. The model is employed for auditing the info for checking integrity of cloud [21] secure dynamic auditing method for data owner is employed this helps to stay far away from the safety and integrity risk of knowledge. New standards for publishing audit data is proposed [22] here various challenges and directions of auditing are discussed. Hence these are the varied related works on key management and auditing.

Various arrangements might be utilized to trade scrambled information with a cloud supplier in a protected way, to such an extent that the cloud supplier isn't straightforwardly depended with key material, evaluating is conveyed for guaranteeing accessibility of information. The fundamental impediment of an open key administration framework, for example, RSA [2] (which represents the creators Rivest, Shamir, and Adleman) relies upon the trouble of calculating enormous whole numbers. High traffic cost is another issue since the single key must be imparted to every single approved client. Clients may join and leave the approved client set every now and again, prompting steady key re-age and redistribution through extra correspondence meetings to deal with client denial; in an exceptionally versatile framework, such occasions may happen at moderately high recurrence. Remote correspondence, is costly and brings about fast battery channel, particularly when transmitting [3].

Scrambled information ought to in a perfect world be put away in the cloud with the goal that it can't be gotten to by cloud supplier. This idea is reliant on the keys being safely overseen by an element outside of the supplier's area. The trouble emerges when new clients join the framework, and existing ones leave, requiring new keys to be created. The scrambled information ought to in a perfect world be changed to such an extent that it might be opened with new keys, without a middle unscrambling step that would permit the cloud supplier to peruse the plaintext; this procedure is known as information re-encryption. In spite of the fact that it gives off an impression of being a promising method in overseeing encoded information as access rights develop after some time, current arrangements in the writing don't address the issue of high versatility to an adequate and good degree; nor do they essentially endeavor to diminish the computational and correspondence trouble on clients associating with the cloud from asset obliged cell phones. The method of Certificate Authority Based Encryption (CABE) [4] offers various focal points. It permits a client to get access to encoded information in the cloud dependent on the ownership of specific properties that fulfill an entrance structure characterized in the cloud, as opposed to the ownership of a key that must be spread to every single invested individual ahead of time. The imperative properties might be controlled by an information proprietor ahead of time; this proprietor is answerable for creating the client information to be shared, encoding it, and transferring it to the cloud. The proprietor may not really be required in each read exchange. Typically, a plan dependent on CABE depends upon the information proprietor conceding access consent through an entrance tree, which requires their steady accessibility. 
In certain works, key material is conveyed among different gatherings; for example, an information proprietor and a believed authorizer may work in show to concede get to consent to different clients [5]; the arrangement, be that as it may, isn't customized for a versatile domain because of its computational requests, the necessary steady accessibility of the information proprietor, and time sensitive termination of access prompting continuous key recovery. Repudiation of an approved client is especially difficult to achieve effectively in CABE and is normally tended to by expanding characteristics or keys with termination dates. A tree of revocable ascribes may should be kept up and a believed party appointed to approve renouncement status. An instrument utilizing direct mystery sharing and paired tree methods is one model [6], yet versatile clients need to bring about the correspondence cost of ceaselessly mentioning new keys. Additionally, the information proprietor is commonly likewise a versatile client, and along these lines can't oversee get to control on interest for all because of its transient network. Disavowal has been suggested that depends on stateless key circulation and access control on the characteristic level [7], however requires a confided in power and hinders the proprietor with a cryptographic blending activity that is computationally exorbitant. High leveled Identity Based Encryption (HIBE) and CABE are joined in another related work [20], to appropriate client keys progressive space experts are utilized; this is done at the expense of expanded stockpiling necessities for key material held by clients and while creating cipher text more prominent measure of handling is done. Dynamic elliptic bend encryption conspire has been proposed for believed information sharing [8]. Be that as it may, it relies on an author transferring scrambled information to the cloud, at that point to perform re-encryption certifications are conveyed to the cloud, and furthermore to the per user on every datum get to endeavor; it is unfeasible when applied to asset obliged cell phones and systems. A plan has been proposed to deal with repudiation in a versatile framework, which uses cloud supplier for disseminating bits of key material and for programmed Certificate Authority Based Encryption [9]; in light of client personality it requires get to exclusively this is one of the constraint right now.

One strategy is to re-scramble the put away substance during recovery. Such a procedure has been applied to an encoded document stockpiling framework where a substance proprietor scrambles squares of substance with exceptional, symmetric substance keys, and these keys are then additionally scrambled to shape a lockbox [10]; clients speak with an entrance control server to unscramble them. The issue is that the substance proprietor oversees get to control for every single other client, which is an extraordinary weight, and requires dynamic re-encryption of similar information at whatever point various clients get to it. In the model thus, once Certificate Authority Based Encryption only happens at whatever point participation changes, apparently a less successive event than that of information get to. Different methodologies require a confided in intermediary for every unscrambling [11], which builds the correspondence cost. Intermediary re-encryption has additionally been joined with CP-ABE [12] to such an extent that re-encryption keys are processed by the cloud supplier dependent on a mystery that is pre shared between the information proprietor and the supplier, just as the supplier's inside clock. The re-encryption keys must be figured for all traits in the entrance structure, which could be very numerous. Another thought is to safely install the information key inside the header of the record put away in the cloud [13]; an advantaged chief gathering is answerable for age of re-encryption keys, however it should likewise circulate the mystery header key to the beneficiary to finish the procedure.

Quality renouncement approach has been recommended, and accordingly, an authority rethinks ace key segments for the properties, client mystery keys are refreshed, and intermediary server re-encodes the information [14]; the disavowal is needy upon changes to traits this make it troublesome, bringing about exorbitant key reports on every repudiation. A method that joins $\mathrm{CP}-\mathrm{ABE}$ with intermediary re-encryption [15] doesn't give off an impression of being profoundly proficient for portable clients: for example, the unscrambling procedure requires handling two access sub trees rather than one.

\section{METHODOLOGY}

System model consist of the architecture diagram of the entire work. This explains clearly what the proposed work is.

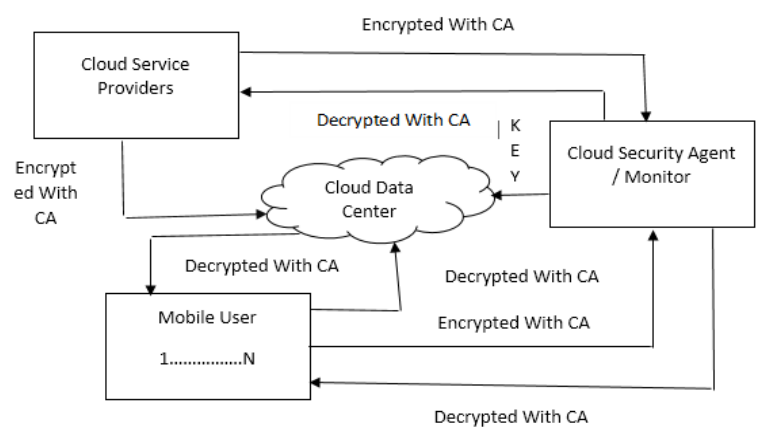

Figure 1. CA based Cryptography Architecture Diagram

\section{Cloud Infrastructure}

The public cloud is used here for storing the details of the mobile users. Public cloud allows system and services to be easily accessible to the general public. Public cloud may be less secure because of its openness. Reencryption done in the cloud provides additional security to the data.

Cloud service providers and manager

Cloud manager provides key for encryption, reencryption and decryption. Manager is a trusted party. The mobile users request keys for encryption, manager also 


\section{Develope An Certificate Authority Based Encryption Cryptography Model For Mobile Applications In Cloud Computing}

Available at https://jscer.org

generates keys for re-encryption which is performed in the cloud.

Mobile based users

Mobile users may be Google's Android, RIM Blackberry, Apple iOS, etc., in this work android mobile users are selected. Android users encoded their data and then upload their data to the cloud. They can also decrypt the data when they need it.

Cloud security monitor

Cloud solutions has got to be auditable so as to enable continuous evaluation of whether the safety level of a cloud supplier's specific solution is sufficient to be used for a given system or solution. At an equivalent time, a cloud supplier must be ready to provide adequate information about audit and assurance to satisfy the customers' risk assessment and be compliant with legislation. For auditing a protocol is been used.

\section{ALGORITHM FOR CRYPTOGRAPHY DEVELOPEMENT}

The technique used is Cipertext-policy attribute based encryption were the encryption is done according to the attribute of the mobile user under this technique symmetric key encryption such as AES is used for encryption, re-encryption and decryption string matching algorithm is used for cloud auditing.

\section{A. AES encryption:}

AES encryption consist of four different stages one of permutation and three of substitution: substitute bytes, shift rows, mix columns and add round key are the various stages in the encryption. The same steps are carried for the reencryption. $C=E_{n}(M)$ Where, $C$ is cipher text; $E$ is encryption, $\mathrm{m}$ is the original message, $\mathrm{En}$ is key for encryption. This is how the encryption takes place.

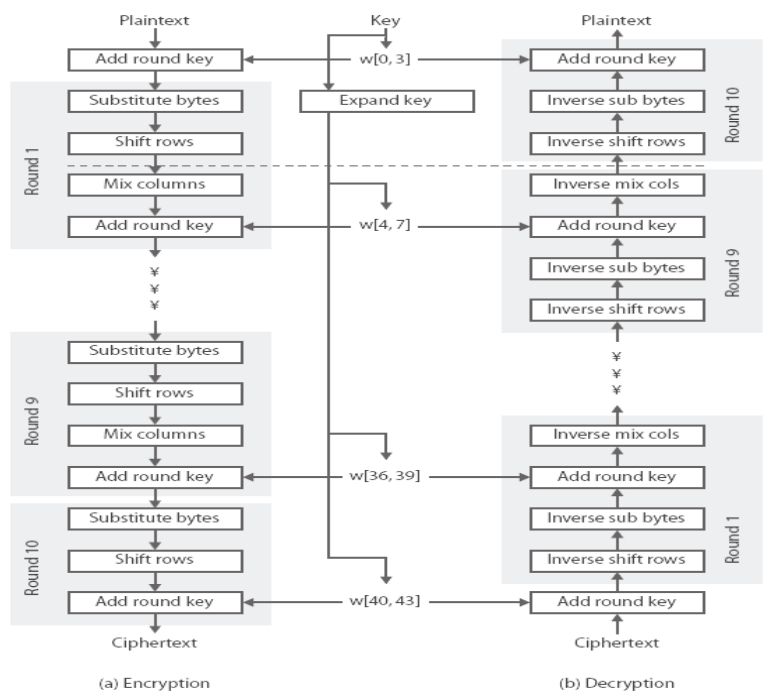

Figure 2 AES structure

The following algorithm shows the step by step process for encryption in AES:

Step 1: Start with the plain text

Step 2: Derive the n no of round keys from the cipher key.

Step 3: Initialize the state array with the block data and add the initial round key to the starting state array.

Step 4: Perform various rounds of state manipulation.

Step 5: Perform the final round of state manipulation.

Step 6: Copy the final state array out as the encrypted data (cipher text), the cipher text is again given for the Certificate Authority Based Encryption process.

Step 8: The same steps are followed for the Certificate Authority Based Encryption process.

\section{B. AES decryption:}

AES decryption is the process of transforming information to make it readable to only those possessing special knowledge, usually referred to as a key decryption is not identical to encryption since steps done in reverse this has inverse shift rows, inverse sub bytes, and inverse mix columns and add round key.

$$
\mathrm{D}_{\mathrm{k}}(\mathrm{C})=\mathrm{D}_{\mathrm{k}}\left(\left(\mathrm{E}_{\mathrm{k}}(\mathrm{m})\right)=\mathrm{m}\right.
$$

Where, $\mathrm{Dk}$ is the key for decryption, $\mathrm{m}$ is the original message, $\mathrm{C}$ is the cipher text. This is how the decryption process takes place.

The following algorithm shows the step by step process for decryption in AES:

Step 1: Start with the cipher text which has been reencrypted in the cloud.

Step 2: Derive the set of round keys and Round 1 is started which consist of inverse sub bytes, inverse shift rows and inverse mix columns and round key.

Step 3: Several rounds of operations are performed. Final round consist of inverse sub bytes, inverse shift rows and round keys alone

Step 4: The plain the final output from the decryption algorithm.

C. String Matching Algorithm for CA security: 
The context of the problem is to find out whether one string is contained in another string. This problem correspond to a part of more general one, called "pattern recognition". The strings considered are sequences of symbols, and symbols are defined by an alphabet. The size and other features of the alphabet are important factors in the design of string-processing algorithms. So this technique is used for auditing whether any other user is using their data.The following algorithm shows the step by step process of string matching algorithm:

Step 1: Select the data to be audited, the patterns will be searched

Step 2: Next counter will be given to move to the next string, this will be carried for the entire pattern of data.

Step 3: If there is any mismatch then the data is altered or any of vulnerabilities are available, if the pattern are correct then the data are safe.

\section{EXPERIMENTAL RESULTS AND DISCUSSIONS}

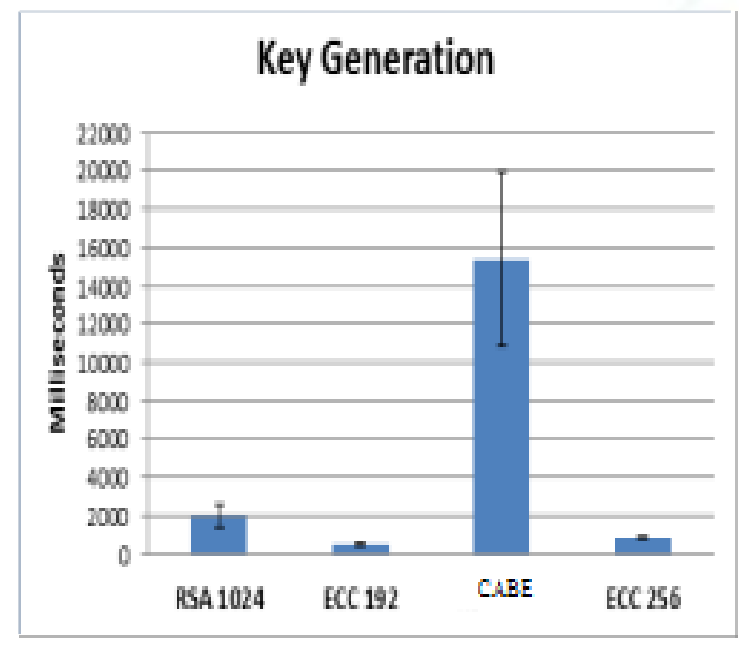

Figure 3 Comparative CABE Key Generation

The proposed methodology has been done in public cloud using different operating system software. Here the AES algorithm is used for encryption, Certificate Authority Based Encryption (CABE) and decryption this ensures security of mobile users data stored in data. String matching algorithm is used for cloud auditing this is used for ensuring data integrity and availability. In the proposed methodology Certificate Authority Based Encryption provides high security. Here the manager provides the key for encryption process, then the mobile users use this key for encrypting the data and then it uploads the data. Here the Certificate Authority Based Encryption (CABE) encryption keys are provided by the cloud manager and Certificate Authority Based Encryption (CABE) is done in the cloud environment itself. The manager provides key for decryption and the user decrypts the data from the cloud environment. Cloud auditing is done regularly for ensuring availability this is the novel modification in the proposed work.

\section{CONCLUSION}

Secure mobile applications in cloud is implemented using Certificate Authority Based Encryption (CABE) technique. Symmetric key encryption is used for encryption, Certificate Authority Based Encryption (CABE) and decryption. Protocol for cloud audit is proposed for ensuring cloud integrity and availability. This work carried in the public cloud in future in it can be done in private or hybrid cloud.

\section{REFERENCES}

[1] P.K. Tysowski and M.A.Hasan, "Hybrid Attribute- Based Encryption and Re-Encryption for Scalable Mobile Applications in Clouds," Technical Report 13, Centre for Applied Cryptographic Research (CACR), Univ. of Waterloo, 2013.

[2] R.L.Rivest, A.Shamir,and L.Adleman, "A Method for Obtaining Digital Signatures and Public-Key Cryptosystems," Comm. ACM, vol. 26, no. 1, pp. 96-99, Jan. 1983.

[3]N.Balasubramanian,A. Balasubramanian, and A.Venkataramani, "Energy Consumption in Mobile Phones: A Measurement Study and Implications for Network Applications," Proc. Ninth ACM SIGCOMM Conf. Internet Measurement Conf. (IMC '09), pp. 280293, 2009.

[4]J.Bethencourt, A.Sahai, and B. Waters, "Cipertext- Policy Attribute-Based Encryption," Proc. IEEE Symp. Security and Privacy (SP '07), pp. 321-334, 2007.

[5] A. Tassanaviboon and G. Gong, "OAuth and ABE Based Authorization in Semi-Trusted Cloud Computing: Aauth," Proc. Second Int'l Workshop Data Intensive Computing in the Clouds (DataCloud-SC'11), pp. 41-50, 2011.

[6] X. Liang, R. Lu, and X. Lin, "Ciphertext Policy Attribute Based Encryption with Efficient Revocation," Technical Report BBCR, Univ. of Waterloo, 2011.

[7] J. Hur and D.K. Noh, "Attribute-Based Access Control with Efficient Revocation in Data Outsourcing Systems," IEEE Trans. Parallel and Distributed Systems, vol. 22, no. 7, pp. 12141221, July 2011.

[8] G. Zhao, C. Rong, J. Li, F. Zhang, and Y. Tang, "Trusted Data Sharing over Untrusted Cloud Storage Providers," Proc. IEEE Second Int'l Conf. Cloud Computing Technology and Science (CLOUDCOM'10), pp. 97-103, 2010.

[9]P.K.Tysowski and M.A. Hasan, "Towards Secure Communication for Highly Scalable Mobile Applications in Cloud Computing Systems," Technical Report 33, Centre for Applied Cryptographic Research (CACR), Univ. of Waterloo, 2011.

[10] G. Ateniese, K. Fu, M. Green, and S. Hohenberger, "Improved Proxy Re-Encryption Schemes with Applications to Secure Distributed Storage," ACM Trans. Information and System Security, vol. 9, pp. 1-30, Feb. 2006.

[11] S. Jahid, P. Mittal, and N. Borisov, "EASiER: EncryptionBased Access Control in Social Networks with Efficient Revocation," Proc. Sixth ACM Symp. Information, Computer and Comm. Security (ASIACCS '11), pp. 411-415, 2011.

[12] Q. Liu, G. Wang, and J. Wu, "Clock-Based Proxy ReEncryption Scheme in Unreliable Clouds," Proc.41st Int'l Conf. Parallel Processing Workshops (ICPPW), pp. 304-305, Sept. 2012.

[13] J.-M. Do, Y.-J. Song, and N. Park, "Attribute Based Proxy Re- Encryption for Data Confidentiality in Cloud Computing Environments," Proc. First ACIS/JNU Int'l Conf. Computers, 
Networks, Systems and Industrial Eng. (CNSI), pp. 248-251, May 2011.

[14] S. Yu, C. Wang, K. Ren, and W. Lou, "Attribute Based Data Sharing with Attribute Revocation," Proc. Fifth ACM Symp. Information, Computer and Comm. Security (ASIACCS '10), pp. 261-270, 2010.

[15] Y. Ming, L. Fan, H. Jing-Li, and W. Zhao-Li, "An Efficient Attribute Based Encryption Scheme with Revocation for Outsourced Data Sharing Control," Proc. First Int'l Conf. Instrumentation, Measurement, Computer, Comm. and Control, pp. 516-520, 2011.

[16] S. Yu, C. Wang, K. Ren, and W. Lou, "Achieving Secure, Scalable, and Fine-Grained Data Access Control in Cloud Computing," Proc. IEEE INFOCOM'10, pp. 534-542, 2010.

[17]K. Yang and X. Jia, "Attributed-Based Access Control for Multi- Authority Systems in Cloud Storage," Proc. IEEE 32nd Int'l Conf. Distributed Computing Systems (ICDCS), pp. 536-545, 2012.

[18]K. Yang, X. Jia, K. Ren, and B. Zhang, "DAC- MACS: Effective Data Access Control for Multi- Authority Cloud Storage Systems," Proc. IEEE INFOCOM, pp. 2895-2903, 2013.

[19] J. Wang, "Java Realization for Cipertext-Policy AttributeBased Encryption," http://github.com/wakemecn, 2012.

[20] G. Wang, Q. Liu, and J. Wu, "Hierarchical Attribute- Based Encryption for Fine-Grained Access Control in Cloud Storage Services," Proc. 17th ACM Conf. Computer and Comm. Security (CCS '10), pp. 735-737, 2010.

[21] S. V. Marshal, "Secure Audit service by using TPA for Data Integrity in Cloud System" International Journal of Innovative Technology and Exploring Engineering (IJITEE), September 2013. [22] H. Rasheed, "Auditing for Standards Compliance in the Cloud: Challenges and Directions" The International Arab Journal of Information Technology, July 2003. 